\title{
Differential DNase I hypersensitivity reveals factor-dependent chromatin dynamics
}

\author{
Housheng Hansen He, ${ }^{1,2,5}$ Clifford A. Meyer, ${ }^{1,5}$ Mei Wei Chen, ${ }^{2,3}$ V. Craig Jordan, ${ }^{4}$ \\ Myles Brown, 2,3,6 and X. Shirley Liu' ${ }^{1,3,6}$ \\ ${ }^{1}$ Department of Biostatistics and Computational Biology, Dana-Farber Cancer Institute and Harvard School of Public Health, Boston, \\ Massachusetts 02115, USA; ${ }^{2}$ Department of Medical Oncology, Dana-Farber Cancer Institute and Harvard Medical School, Boston, \\ Massachusetts 02115, USA; ${ }^{3}$ Center for Functional Cancer Epigenetics, Dana-Farber Cancer Institute, Boston, Massachusetts 02215, \\ USA; ${ }^{4}$ Department of Oncology, Lombardi Cancer Center, Georgetown University Medical Center, Washington, DC 20057, USA
}

Transcription factor cistromes are highly cell-type specific. Chromatin accessibility, histone modifications, and nucleosome occupancy have all been found to play a role in defining these binding locations. Here, we show that hormone-induced DNase I hypersensitivity changes ( $\triangle D H S$ ) are highly predictive of androgen receptor (AR) and estrogen receptor 1 (ESRI) binding in prostate cancer and breast cancer cells, respectively. While chromatin structure prior to receptor binding and nucleosome occupancy after binding are strikingly different for ESRI and AR, $\triangle D H S$ is highly predictive for both. AR binding is associated with changes in both local nucleosome occupancy and DNase I hypersensitivity. In contrast, while global ESRI binding is unrelated to changes in nucleosome occupancy, DNase I hypersensitivity dynamics are also predictive of the ESRI cistrome. These findings suggest that AR and ESR1 have distinct modes of interaction with chromatin and that DNase I hypersensitivity dynamics provides a general approach for predicting cell-type specific cistromes.

[Supplemental material is available for this article.]

In eukaryotes, transcription is regulated in a cell-type and condition-specific manner through the association of transcription factors with chromatin. The genome-wide binding sites of transcription factors, or the transcription factor cistromes, are influenced by the active protein levels of the transcription factors, chromatin structure, and DNA sequence. The nucleosome is the fundamental unit of chromatin structure and has been thought to compete with transcription factors for occupancy at thermodynamically favorable genomic loci. By comparing nucleosome occupancy maps generated from nucleosome-resolution H3K4me2 ChIP-seq, we found that nucleosome occupancy changes can be predictive of transcription factor cistromes. In particular, the binding of androgen receptor (AR) in prostate cancer $\mathrm{LNCaP}$ cells leads to an increased occupancy of nucleosomes flanking the AR binding site and decreased nucleosome occupancy in the position of the binding site itself (He et al. 2010). This approach also correctly predicted the binding of two factors, POU2F1 and NKX3-1, which are part of the secondary cellular response to androgens (He et al. 2010). This phenomenon is not unique to the LNCaP AR system; it has also been observed with CDX2, HNF4A, and GATA6 binding in intestinal differentiation (Verzi et al. 2010) and with GATA1 in hematopoiesis (Hu et al. 2011).

DNase I hypersensitivity is an alternative measure of chromatin accessibility (Wu 1980). DNase I hypersensitive sites (DHS), short regions of chromatin that are highly sensitive to cleavage by DNase I, typically occur in nucleosome free regions and frequently arise as a result of transcription factor binding. DNase I digestion followed by high-throughput sequencing (DNase-seq) has evolved into a powerful technique for identifying genome-wide DNase

\footnotetext{
${ }_{6}^{5}$ These authors contributed equally to this work.

${ }^{6}$ Corresponding authors.

E-mail myles_brown@dfci.harvard.edu.

E-mail xsliu@jimmy.harvard.edu.

Article published online before print. Article, supplemental material, and publication date are at http://www.genome.org/cgi/doi/10.1101/gr.133280.111.
}

hypersensitive sites (Ling et al. 2010; John et al. 2011; Siersbaek et al. 2011). Because transcription factor binding sites tend to be DNase I hypersensitive and DNase-seq does not require a factorspecific antibody, DNA sequence motif analysis on DHS data has been proposed as a method for discovering the binding sites of multiple transcription factors in a single experiment (Pique-Regi et al. 2011; Song et al. 2011).

To analyze the effects of androgen receptor (AR) and estrogen receptor 1 (ESR1) binding on DHS, we conducted genome-wide DNase-seq in both unstimulated and hormone-stimulated conditions. Using a quantitative measurement of DHS changes $(\triangle \mathrm{DHS})$ between these conditions, we were able to predict the ESR1 and AR cistromes. Although they are related members of the steroid receptor family, AR and ESR1 display distinct DHS profiles. Binding of both ESR1 and AR are frequently associated with significant increases in DHS signal upon hormone stimulation; however, ESR1 sites show strong DHS prior to binding and AR sites do not. Following hormone stimulation, FOXA1 binding sites that lacked AR or ESR1 binding are associated with a significant decrease in DHS. In MCF-7 cells, this change in DHS is linked not to a change in FOXA1 binding but rather to a decrease in the binding of the ESR1 coactivator, NCOA3, supporting a model of physiologic squelching. This study demonstrates that $\triangle \mathrm{DHS}$ is a more effective and general approach to predict perturbation-induced transcription factor binding sites than either static DHS or nucleosome resolution H3K4me2 ChIP-seq.

\section{Results}

Estrogen receptor binding in breast cancer cells is not associated with significant nucleosome depletion

Based on our earlier work demonstrating the association between AR binding and nucleosome depletion (He et al. 2010), we carried out an H3K4me2 ChIP-seq experiment on MNase digested chromatin in the MCF-7 breast cancer cell line comparing unstimulated 
(Veh) cells with cells grown under conditions of estrogen stimulation (E2). Consistent with previous studies (Barski et al. 2007; He et al. 2010), the H3K4me2 sites in both samples were mainly located in intergenic and intronic regions and were also found to be enriched in promoter regions (Fig. 1A). Over 64\% of estrogen receptor 1 (ESR1) binding sites overlapped with regions enriched in H3K4me2 (estrogen-stimulated) (Fig. 1B). We examined the distribution of H3K4me2 signals relative to the center of all ESR1 binding sites. Although in some cases ESR1 binds to regions depleted of H3K4me2 signal (Supplemental Fig. 1A), in both the vehicle and stimulated conditions the overall pattern shows a peak in the H3K4me2 signal that overlaps with the ESR1 binding sites (Fig. 1C).

We systematically assessed ESR1 binding as a function of the nucleosome stabilization-destabilization (NSD) score, a measure of nucleosome occupancy changes established in previous studies (He et al. 2010). The fraction of ESR1 binding sites located in high NSD scoring regions was no greater than the fraction in regions with low NSD scores (Fig. 1D). This pattern is significantly different from that observed in AR binding (Supplemental Fig. 1B). In AR binding an H3K4me2 tag density peak at the AR binding site becomes a trough after androgen stimulation, resulting in high NSD scoring regions being highly predictive of AR binding (He et al. 2010). Whereas in MCF-7 the distributions of NSD scores at ER and
non-ER sites are not significantly different (Supplemental Fig. 1C, $P$-value $=0.25$ ), the distributions of NSD scores in LNCaP AR and non-AR sites are significantly different (Supplemental Fig. 1D, $P$-value $=2.2 \times 10^{-16}$ ).

In order to determine whether the differences in the behavior of AR in LNCaP cells and ESR1 in MCF7 cells were due to a difference in the transcription factors or the cell lines, we analyzed H3K4me2 enrichment at AR, ESR1, and FOXA1 sites together (Fig. 2A,B; Supplemental Fig. 2A,B). We included the winged helix transcription factor FOXA1 in the analysis as it acts as a "pioneer factor" in breast cancer cells and is required for ESR1 binding to a large proportion of its binding sites (Carroll et al. 2005; Lupien et al. 2008). The role of FOXA1 in AR action in prostate cancer cells is more complex, though a significant number of AR-bound sites are also bound by FOXA1 (Lupien et al. 2008; Wang et al. 2011). Consistent with our previous findings (He et al. 2010), sites bound by FOXA1 alone in either LNCaP or MCF7 cells show a pair of stimulus-independent peaks that flank a trough directly over the FOXA1 binding site (Fig. 2A,B, right panels).

When we examined the H3K4me 2 signal at sites bound by AR or ESR1 that lacked FOXA1, we observed very different patterns. In LNCaP cells, AR binding sites that do not bind FOXA1 had a broad peak of $\mathrm{H} 3 \mathrm{~K} 4 \mathrm{me} 2$ prior to hormone stimulation that resolved into
A

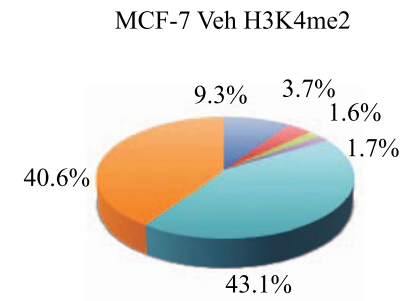

promoter

- exon

- 5'UTR

- 3'UTR

MCF-7 E2 H3K4me2

intron

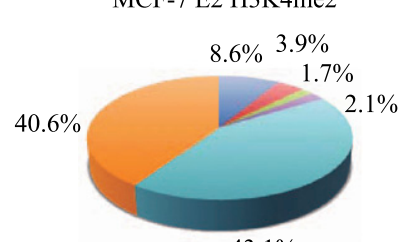

$43.1 \%$

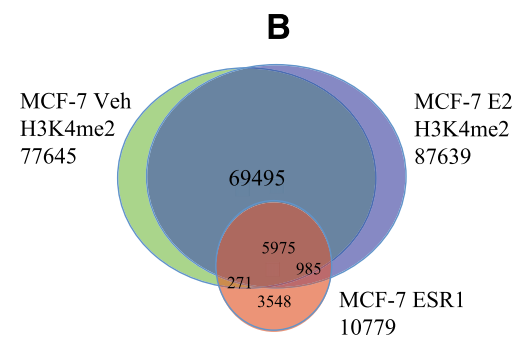

C
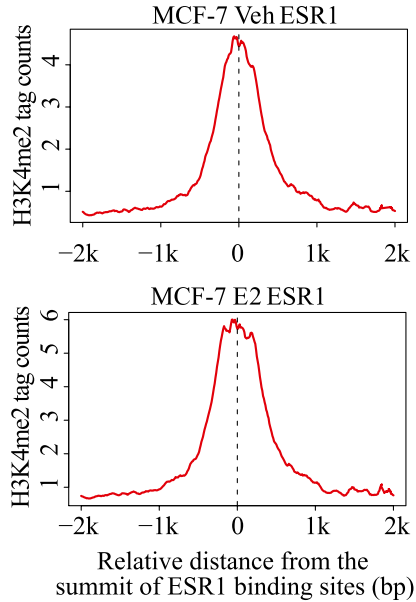

D

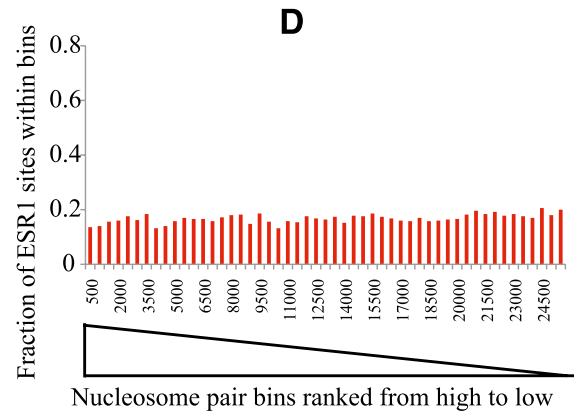

Figure 1. Characteristics of H3K4me2 ChIP-seq in MCF-7 cells. (A) Location of H3K4me2 ChIP-enriched peaks relative to gene annotations in unstimulated (Veh) and estrogen-stimulated (E2) conditions. (B) Venn diagram of ESR1 binding loci in relation to H3K4me2-enriched regions. (C) Distribution of H3K4me2 ChIP-seq signal at non-promoter (>1 kb from TSSs) ESR1 binding sites under unstimulated and estrogen-stimulated conditions. (D) The fraction of ESR1 binding sites in paired nucleosome bins sorted in descending order by NSD score (stimulated vs. unstimulated). Paired nucleosome regions are ranked by the NSD score that represents the differences in the H3K4me2 tag counts before and after estrogen treatment. These ranked regions are grouped into bins of 500 to calculate the proportion of real binding sites as a function of rank. ( $Y$-axis) Fraction of the regions in each bin that overlap with ESR1 ChIP-seq enriched regions. 
two sharp peaks flanking the AR binding site upon AR activation (Fig. 2A, left panels). In contrast, ESR1 binding sites in MCF7 cells that lack FOXA1 had a broad peak of H3K4me2 centered over the ESR1 binding site both before and after ESR1 activation (Fig. 2B, left panels). The pattern of H3K4me2 at the shared AR/FOXA1 and ESR1/FOXA1 sites was also distinct. H3K4me2 signal at the AR/FOXA1 bound sites indicates nucleosome depletion and better positioned flanking nucleosomes after AR activation (Fig. 2A, center panels). In contrast, the pattern at ESR1/FOXA1 sites is similar to the ESR1-unique sites and has a single broad peak both before and after ESR1 activation (Fig. 2B, center panels). NPS, an algorithm that predicts nucleosome position (Zhang et al. 2008b), also predicts clearly different nucleosome distributions relative to ESR1-unique binding sites, FOXA1-unique binding sites, and shared sites (Supplemental Fig. 1C-E). At sites of ESR1 binding with or without FOXA1, the predicted nucleosomes more frequently overlap the ESR1 binding site (Supplemental Fig. 1E,F) while FOXA1 sites that lack ESR1 binding sites have a peak of binding that is in a region removed from a nucleosome center (Supplemental Fig. 1G).

To further test whether the differences between ESR1 and AR are intrinsic to the transcription factors, we examined the MCF-7-derived hormone-independent breast cancer cell line MCF-7:2A (Pink et al. 1995; Ariazi et al. 2011). While MCF7:2A cells grow in the absence of estrogen or androgen, their growth is inhibited by silencing of either ESR1 or AR (data not shown). Sixty-five percent of the ESR1 binding sites in MCF-7 under the E2stimulated condition overlap with those of MCF-7:2A in the absence of estrogen (Supplemental Fig. 2C). While there is significant overlap in the ESR1 and AR binding sites in MCF-7:2A, there are also many ESR1- and AR-unique sites (Fig. 2C, Venn diagram). MNase digested H3K4me2 ChIP-seq in MCF-7:2A was performed, and the distribution of H3K4me2 at ESR1unique, AR-unique, and shared sites was determined (Fig. 2C; Supplemental Fig. 2D). At the ESR1-unique binding sites, H3K4me2 formed a sharp, unimodal peak at the binding site (Fig. 2C, left panel). In contrast, the AR-unique sites are associated with a broader H3K4me2 tag distribution with two modes that flank the AR binding site (Fig. 2C, right panel). Shared ESR1 and AR binding sites had an H3K4me2 profile with an intermediate
A
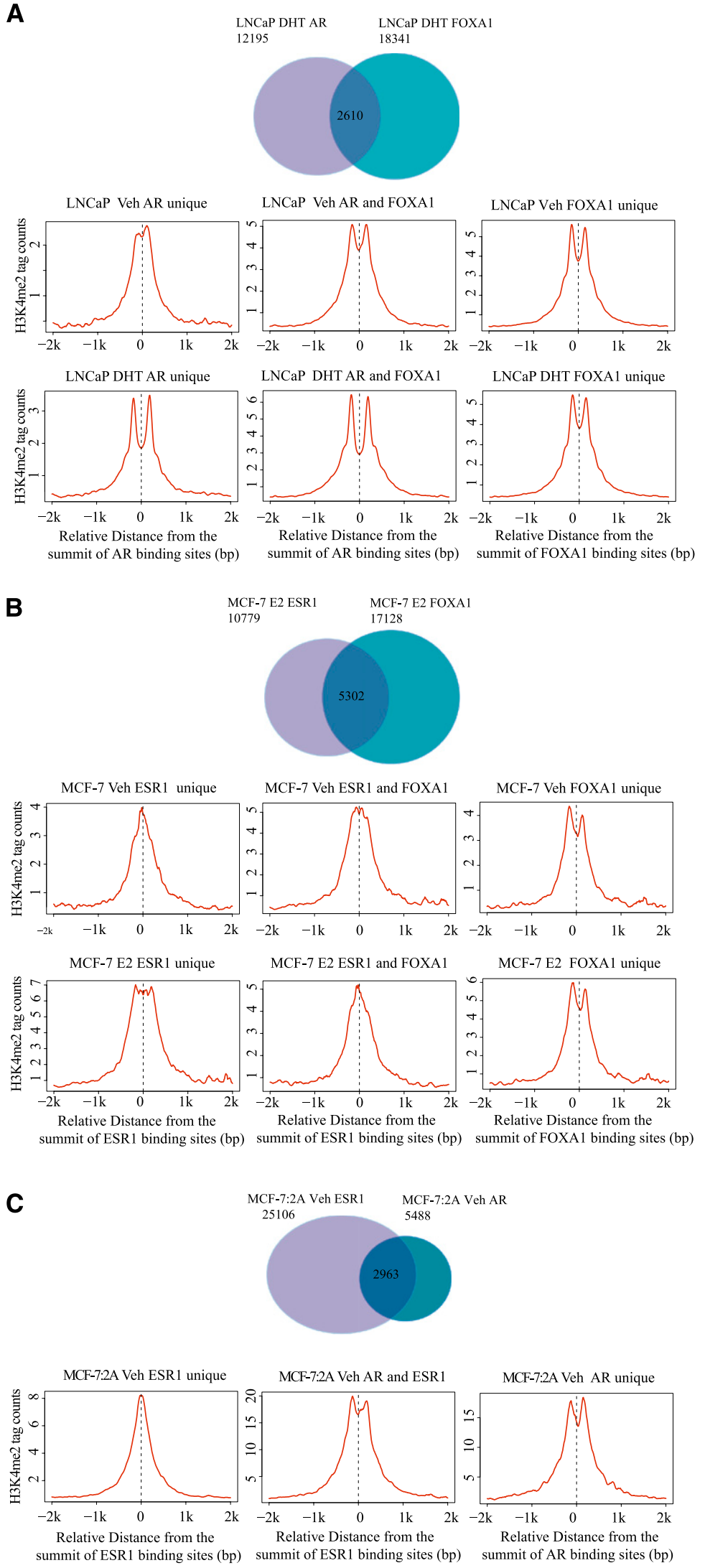

Figure 2. (Legend on next page) 
distribution between that of the ESR1-unique and AR-unique sites (Fig. 2C, middle panel). These results suggest that, although AR binding involves depletion of a nucleosome directly over the AR binding site, ESR1 binding does not.

\section{Quantitative measures of DNase I dynamics are predictive of TF binding}

Given our finding that ESR1 binding could not be predicted by changes in the occupancy of H3K4me2 marked nucleosomes, we investigated stimulus-dependent changes in DNase I hypersensitivity (DHS) as a complement to nucleosome occupancy. An analysis of DHS under unstimulated (Veh) and androgen-stimulated (DHT) conditions in the LNCaP cell line demonstrated that $51 \%$ of AR binding sites overlap with androgen-stimulated DHS regions (Fig. 3A), as would be expected from our prior work on nucleosome occupancy. When we analyzed DHS in MCF-7 cells in unstimulated and estrogen-stimulated (E2) conditions, we found that $\sim 63 \%$ of ESR 1 binding sites overlap with stimulated DHS regions (Fig. 3B). In LNCaP cells, increasing the sequencing depth from $50 \mathrm{M}$ to $70 \mathrm{M}$ increased the proportion of AR sites that overlapped a DHS site from 51\% to 55\% (Supplemental Fig. 3A). Similarly, increasing the sequencing depth in MCF-7 cells from $28 \mathrm{M}$ to $70 \mathrm{M}$ raised the proportion of ESR1 sites that overlap with DHS from $63 \%$ to $71 \%$ (Supplemental Fig. 3B). ESR1 and AR sites that are not associated with DHS show significantly lower levels of binding than those that are associated with DHS (Supplemental Fig. 3C,D).

DHS regions encompass genomic locations that are associated with a variety of transcription factors and other chromatin-associated complexes; therefore, we investigated whether changes in DHS between conditions can be used to enhance the specificity of transcription factor binding site prediction. Starting with the set of DHS regions that were detected under hormone-stimulated conditions, we ranked the regions by three criteria: the DHS tag count in the unstimulated condition, the DHS tag count in the stimulated condition, and a score representing the change in the number of tag counts between the two conditions ( $\triangle \mathrm{DHS}$ ) (Fig. 3C,D). The results for the LNCaP AR and MCF-7 ESR1 systems were quite distinct. In LNCaP cells, the level of DHS is not a strong predictor of AR binding in either the unstimulated or stimulated condition, although in both cases it is somewhat informative. In contrast, the change in DHS, $\triangle \mathrm{DHS}$, is a very strong predictor of AR binding (Fig. 3C). Interestingly, in the MCF-7 system, the level of DHS under unstimulated conditions is slightly predictive of ESR1 binding; however, estrogen-stimulated DHS and, most significantly, $\triangle \mathrm{DHS}$ are progressively superior at predicting ESR1 binding (Fig. 3D). These results suggest on a genome-wide scale that at AR and ESR1 binding sites DHS increases upon receptor binding.

On a genomic scale, DNA sequence recognition motifs alone are poor predictors of in vivo ESR1 and AR binding. However, within DHS regions, DNA sequence motifs may be useful for iden- tifying the DHS sites associated with the binding of a particular transcription factor. Starting with the set of DHS regions detected in the hormone-stimulated condition, we ranked the regions by three criteria: $\triangle D H S$, strength of the AR or ESR1 DNA sequence motif, and a combination of the sequence motif and $\triangle \mathrm{DHS}$. In both the LNCaP and MCF7 systems, the nuclear receptor binding motifs are capable of discerning the binding locations of the specific factors from the remainder of the open chromatin regions (Supplemental Fig. 4A,B). Therefore, while DNA sequence motifs may not be reliable predictors of transcription factor binding across the entire genome (Carroll et al. 2006), they are reliable predictors within the regions of open chromatin. The best prediction of AR or ESR1 binding, however, was obtained by combining $\triangle \mathrm{DHS}$ and motif based rankings. To further assess the ability of our approach to predict genome-wide receptor binding sites, we carried out precision-recall analysis for ESR1 (Fig. 3E) and AR (Supplemental Fig. 5). Precision is the fraction of predicted binding sites that are true positives and recall is the fraction of true binding sites identified. As seen for ESR1 in MCF-7 cells, DNA sequence motif alone is a poor predictor of binding. Combining static DHS peaks with motif yields a significantly better prediction, while combining $\Delta \mathrm{DHS}$ with motif is most predictive. Interestingly when we plotted the precision-recall value for the ESR1 binding sites predicted by the CENTIPEDE algorithm (Pique-Regi et al. 2011) we found a point-prediction (see Methods) that is very similar to what we find using static DHS plus motif. Thus $\Delta$ DHS plus motif provides a powerful computational model for TF binding site prediction.

\section{DNase I hypersensitivity is dependent on combinations of bound transcription factors}

We further investigated the influence of combinations of ESR1 and AR binding with FOXA1 on $\triangle \mathrm{DHS}$. We found that the majority of FOXA1 sites are DHS in the LNCaP (72\%) and MCF-7 (64\%) cell lines (Supplemental Fig. 6). Interestingly, while DHS tends to increase at shared nuclear receptor FOXA1 sites, FOXA1 sites that do not overlap with AR or ESR1 loci after stimulation are associated with a decrease in DHS (Fig. 3F,G). In addition, $\triangle \mathrm{DHS}$ at nuclear receptor binding loci are modified by the presence of FOXA1 in a cell line dependent fashion. In MCF-7, ESR1 sites that overlap with FOXA1 loci tend to show larger increases in DHS than the non-FOXA1 binding site containing ESR1 sites (Fig. 3G). In contrast, we observe a larger $\triangle \mathrm{DHS}$ in non-FOXA1 AR binding sites than in the AR sites that overlap with FOXA1 (Fig. 3F) in LNCaP cells, despite the fact that the hormone-stimulated DHS signals in both cell lines are greatest at the shared nuclear receptor-FOXA1 shared sites (Supplemental Fig. 7).

\section{Coactivator activity is detected by $\Delta \mathrm{DHS}$}

One motivation for generating cistromes is to gain insight into the regulation of gene expression. To determine if $\Delta \mathrm{DHS}$ can inform transcriptional regulation, we compared published LNCaP gene expression data (Wang et al. 2009) and MCF-7 GRO-seq data (Hah et al. 2011) with three sets of DHS sites: hormoneincreased (top $5000 \Delta \mathrm{DHS}$ ), hormonediminished (bottom $5000 \Delta \mathrm{DHS}$ ), and hormone-unchanged (middle $5000 \Delta \mathrm{DHS}$ ). In both LNCaP and MCF-7 cells, the ratio of up-regulated genes to non-regulated genes (odds ratio) has a strong positive association with the hormone-increased DHS sites within $20 \mathrm{~kb}$ of the transcription start site 
(TSS) (Fig. 4A, red bars). In contrast, there is no positive association between hormone-unchanged or -diminished DHS sites with increased gene expression (Fig. 4A, blue and green bars).

A

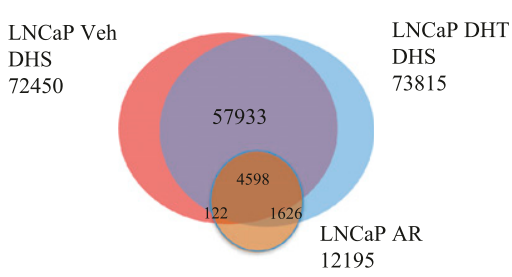

B

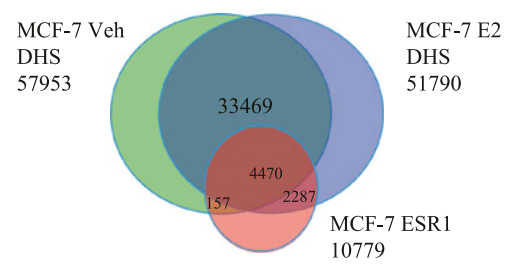

D

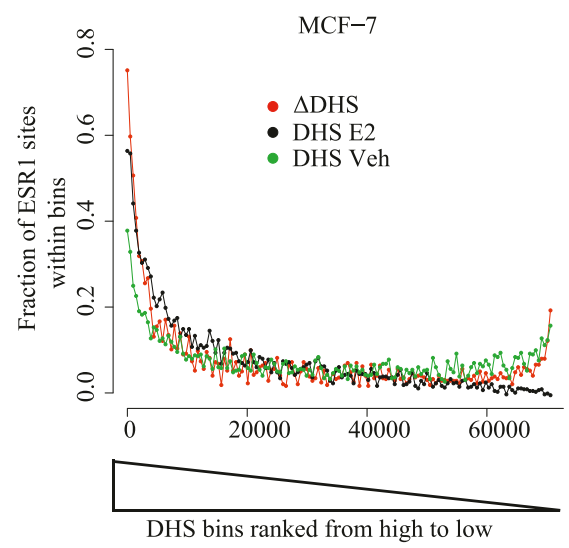

$\mathbf{F}$

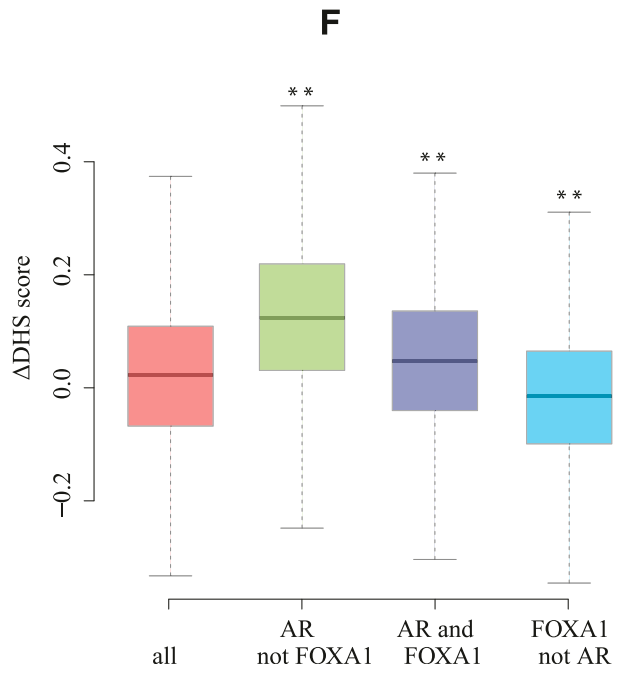

We have previously shown using ESR1 ChIP-chip and gene expression microarrays in MCF-7 that early up-regulated genes, which increased after $3 \mathrm{~h}$ of hormone stimulation, are strongly associated

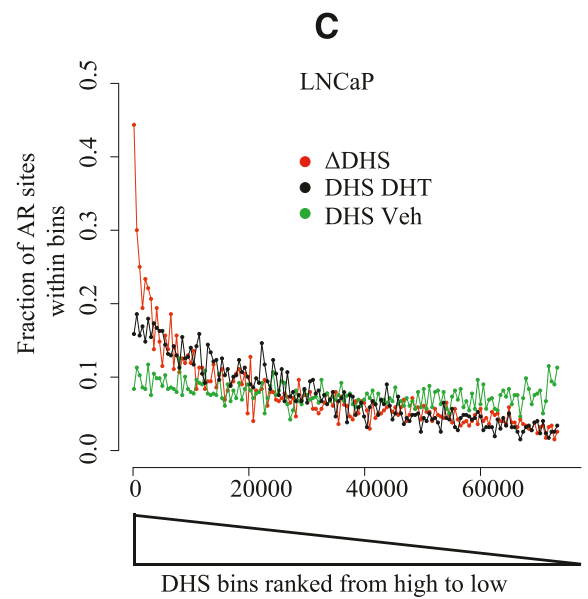

E

MCF-7

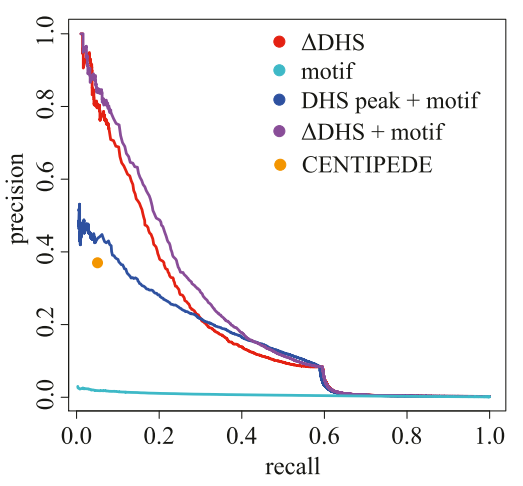

G

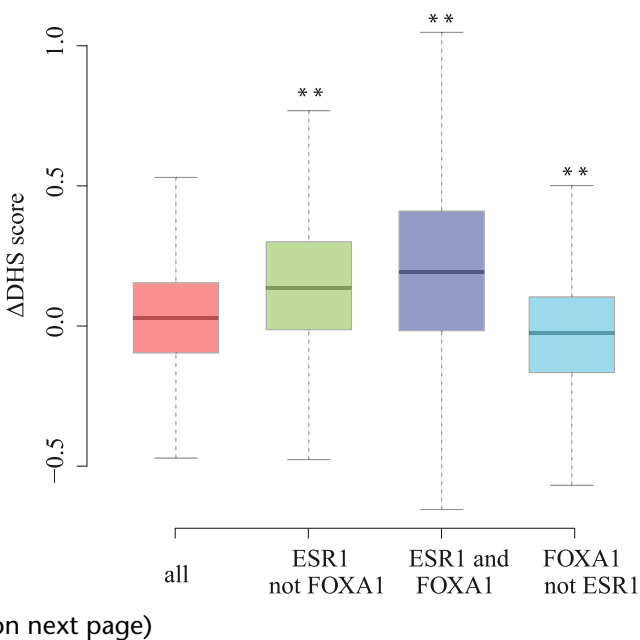


with ESR1 binding, whereas the early down-regulated genes are not (Carroll et al. 2006). These findings were confirmed by Hah and colleagues using GROseq (Hah et al. 2011). Interestingly, we find a strong association of early downregulated genes with the hormonediminished DHS sites (Fig. 4B, green bars). Motif analysis shows that, while the hormone-induced DHS regions are enriched for motifs for ESR1, forkhead and AP-1, the hormone-diminished DHS sites are enriched primarily for the forkhead motif and not the ESR1 motif (Table 1). We confirmed that FOXA1 binding is enriched at the sites with both the highest and lowest $\triangle$ DHS using FOXA1 ChIP-seq data (Fig. 5A). Interestingly, the FOXA1 sites lacking ESR1 are only strongly associated with the sites with the lowest $\Delta$ DHS (Fig. 5B). One explanation for these findings would be that, at sites where FOXA1 is bound in the absence of ESR1, FOXA1 binding is reduced upon estrogen stimulation.

To investigate whether FOXA1 sites without ESR1 binding have reduced enrichment upon stimulation, we compared the FOXA1 ChIP-seq reads under vehicle and stimulated conditions (Joseph et al. 2010) within the three categories of 5000 DHS sites (Fig. 5C). Starting with DHS regions detected in the E2-stimulated condition we counted the number of FOXA1 tags obtained from ChIP-seq in unstimulated and E2-stimulated conditions. If we restrict the set of DHS regions to include only the middle 5000 hormone-unchanged regions and plot the FOXA1 tag count for the stimulated condition as a function of that for the unstimulated condition, we see a linear trend, represented by the blue regression line in Figure 5C. In a similar way if we select the top 5000 hormone-increased DHS sites, we again see a linear trend but the slope of the regression line (red) for this trend is greater. This indicates that there is more hormonestimulated FOXA1 binding in the hormone-increased set than in the hormone-unchanged set. If we select the top 5000 hormonediminished sites and plot a regression line (green), we see the slope of the regression line through the hormone-diminished set is not significantly lower than that of the hormone-unchanged set. A reulated genes $(B)$.
A

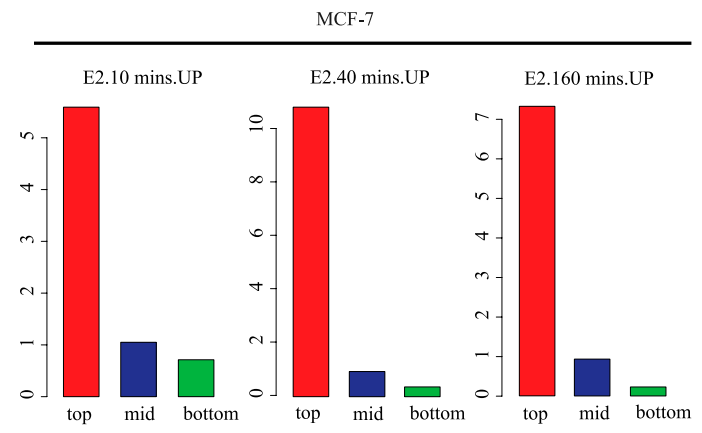

B

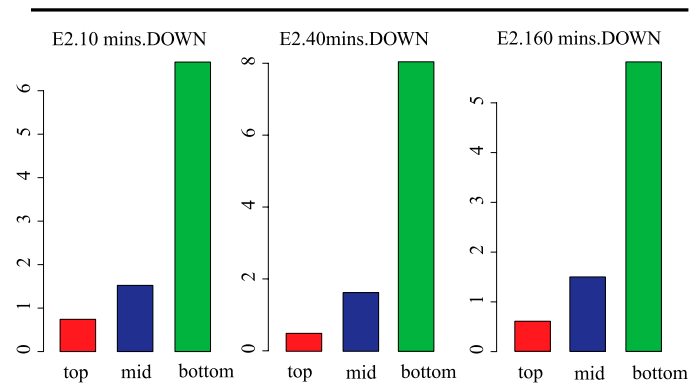

Figure 4. Association between dynamic DNase-seq and differentially expressed genes. Three groups of DHS are represented in LNCaP and MCF-7 cells: hormone-induced DHS sites (red); hormone-unchanged DHS sites (blue); and hormone-diminished DHS sites (green). ( $Y$-axis) Odds ratio calculated by he following formula: (up-regulated genes with at least one nearby site/non-regulated genes with at site). In this definition, "nearby" means within $20 \mathrm{~kb}$ of the TSS. The hormone-induced sites are associated with up-regulated genes $(A)$, while the hormone-depleted sites are associated with down-reg-

duction in FOXA1 binding does not, therefore, appear to explain the decrease in DHS.

Physiological squelching (Meyer et al. 1989) has been postulated to be an important mode of early estrogen down-regulation (Carroll et al. 2006). This phenomenon occurs when multiple factors in the same cell share a common factor, such as a coactivator that is present at a limiting concentration. The transcription factors interfere with each other, "squelching" each other's influence. Of the numerous known ESR1 coactivators, NCOA3 has been shown to have a particularly strong synergy with ESR1 in enhancing gene expression (Torchia et al. 1997). As with FOXA1, NCOA3 binding was associated with both the highest and lowest $\triangle \mathrm{DHS}$ sites overall and with only the lowest $\Delta$ DHS sites at loci lacking ESR1 (Supplemental Fig. 8).

Figure 3. Characteristics of DNase I hypersensitivity sequencing. ( $A$ ) Venn diagram of the DHS and AR peaks in LNCaP. The DNase-seq sequencing depth was normalized to the lower sequencing depth for the unstimulated (50 M) and androgen-stimulated (70 M) conditions. ( $B$ ) Venn diagram of the DHS and ESR1 peaks in MCF-7. DNase-seq sequencing depth was normalized to the lower sequencing depth of the unstimulated (28 M) and estrogen-stimulated (70 $\mathrm{M})$ conditions. $(C, D)$ The fraction of LNCaP AR $(C)$ or MCF-7 ESR1 $(D)$ binding sites in bins ranked by three measures: DNase-seq tag counts in stimulated and unstimulated conditions and a score, $\triangle \mathrm{DHS}$, representing the change in DNase I hypersensitivity between the two conditions. The DNase-seq peak regions under the stimulated condition are ranked by these measures. To calculate the proportion of real binding sites as a function of rank, these ranked regions are grouped into bins of 500. ( $Y$-axis) Fraction of regions in each bin that overlap with AR $(C)$ or ESR1 $(D)$ ChIP-seq enriched regions. ( $E$ ) The precision-recall curves for prediction power of MCF-7 ESR1 binding sites were calculated by five measures: $\Delta$ DHS, ESR1 motif, ESR1 motif in E2 DHS, sqrt([ $\Delta D H S$ rank] ${ }^{*}[$ motif rank]), and results generated by the CENTIPEDE algorithm on ENCODE MCF-7 DNase-seq data (see Methods). ( $F, G)$ Box plots showing the distribution of the DNase-seq change ( $\triangle$ DHS) between the unstimulated and stimulated conditions in LNCaP $(F)$ and MCF-7 (G) cells. "All" represents all the DHS sites in MCF-7 and LNCaP; "AR not FOXA1" and "ESR1 not FOXA1" represent AR and ESR1 binding sites that do not overlap with FOXA1; "AR and FOXA1" and "ESR1 and FOXA1" represent AR and ESR1 binding sites that overlap with FOXA1; "FOXA1 not AR" and "FOXA1 not ESR1" represent FOXA1 binding sites that do not overlap with AR and ESR1. (**) Wilcoxon rank-sum test $P$-values $<0.01$, comparing "all" with the other categories. 
Table 1. Top 20 motifs enriched in the top 5000 and bottom 5000 MCF-7 $\Delta$ DHS regions

\begin{tabular}{|c|c|c|c|c|c|c|c|}
\hline \multicolumn{4}{|c|}{ Top $5000 \Delta \mathrm{DHS}$} & \multicolumn{4}{|c|}{ Bottom $5000 \Delta \mathrm{DHS}$} \\
\hline Motif ID & Gene symbol & Number of hits & $P$-value & Motif ID & Gene symbol & Number of hits & $P$-value \\
\hline M00959 & ESR1 & 2002 & $1.00 \times 10^{-30}$ & M00724 & FOXA1 & 3806 & $1.00 \times 10^{-30}$ \\
\hline M00515 & PPARG & 997 & $1.00 \times 10^{-30}$ & M00131 & FOXA2 & 3782 & $1.00 \times 10^{-30}$ \\
\hline M00925 & JUN/FOS & 1557 & $1.00 \times 10^{-30}$ & M01012 & FOXM1 & 4283 & $1.00 \times 10^{-30}$ \\
\hline M00156 & RORA & 2797 & $1.00 \times 10^{-30}$ & M00269 & FOXA2 & 4803 & $1.00 \times 10^{-30}$ \\
\hline M00037 & NFE2 & 1696 & $1.00 \times 10^{-30}$ & M00292 & FOXD1 & 3250 & $1.00 \times 10^{-30}$ \\
\hline M00285 & NFE2L1 & 4634 & $1.00 \times 10^{-30}$ & M00422 & FOXJ2 & 4171 & $1.00 \times 10^{-30}$ \\
\hline M00495 & $B A C H 1$ & 972 & $1.00 \times 10^{-30}$ & M00290 & FOXF2 & 4927 & $1.00 \times 10^{-30}$ \\
\hline M00490 & $\mathrm{BACH} 2$ & 875 & $1.00 \times 10^{-30}$ & M00291 & FOXC1 & 4891 & $1.00 \times 10^{-30}$ \\
\hline M00239 & NR1D1 & 1531 & $1.00 \times 10^{-30}$ & M00289 & FOXI1 & 4747 & $1.00 \times 10^{-30}$ \\
\hline M00727 & SF1 & 4133 & $1.00 \times 10^{-30}$ & M00266 & CROCC & 4693 & $1.00 \times 10^{-30}$ \\
\hline M00511 & SLC7A1 & 3291 & $1.00 \times 10^{-30}$ & M00268 & XFD2 & 4962 & $1.00 \times 10^{-30}$ \\
\hline M01138 & ROR1 & 3723 & $1.00 \times 10^{-30}$ & M01137 & $\mathrm{FOXO} 3$ & 4496 & $1.00 \times 10^{-30}$ \\
\hline M00292 & FOXD1 & 4559 & $1.00 \times 10^{-30}$ & M00809 & FOX factors & 3571 & $1.00 \times 10^{-30}$ \\
\hline M00157 & ROR2 & 1568 & $1.00 \times 10^{-30}$ & M00472 & FOXO4 & 4543 & $1.00 \times 10^{-30}$ \\
\hline M00204 & GCN4 & 808 & $1.00 \times 10^{-30}$ & $\mathrm{M} 00742$ & FOXJ1 & 4768 & $1.00 \times 10^{-30}$ \\
\hline M00821 & NFE2L2 & 2809 & $1.00 \times 10^{-30}$ & $\mathrm{M} 00267$ & XFD1 & 4583 & $1.00 \times 10^{-30}$ \\
\hline M00035 & $M A F$ & 2896 & $1.00 \times 10^{-30}$ & M00951 & GRHL3 & 3561 & $1.00 \times 10^{-30}$ \\
\hline M00269 & FOXA2 & 2247 & $2.06 \times 10^{-27}$ & M00294 & FOXF1 & 4859 & $1.00 \times 10^{-30}$ \\
\hline M00724 & FOXA1 & 4452 & $1.34 \times 10^{-25}$ & M00475 & FOXO3 & 4259 & $1.00 \times 10^{-30}$ \\
\hline M00983 & $M A F$ & 949 & $2.89 \times 10^{-25}$ & M00473 & FOXO1 & 4800 & $1.00 \times 10^{-30}$ \\
\hline
\end{tabular}

Using published MCF-7 NCOA3 ChIP-seq data (Joseph et al. 2010; Lanz et al. 2010), we compared NCOA3 and FOXA1 cistromes, finding $61 \%$ of FOXA1 binding sites overlap with NCOA3 (Supplemental Fig. 9). Analyzing the three categories of DHS sites using this NCOA3 ChIP-seq data, we found that NCOA3 binding associated with hormone-diminished DHS loci was distributed in a clearly distinct pattern from the hormone-unchanged sites (Fig. 5D). The slope of the regression line of the hormone-diminished set was significantly lower than that of the hormone-unchanged set (Fig. 5D). As ESR1 directly interacts with NCOA3, these data support the hypothesis that ESR1 competes with FOXA1 for limited amounts of NCOA3 that are either directly associated with FOXA1 or associated with other transcription factors whose binding is facilitated by FOXA1.

If physiological squelching is responsible for the E2-stimulated loss of NCOA3 at FOXA1 binding sites, then higher concentrations of NCOA3 in the nucleus should result in a reduced E2stimulated NCOA3 loss. We tested this by overexpressing NCOA3 (Supplemental Fig. 10A), selecting six FOXA1 non-ESR1 binding sites from hormone-diminished DHS and determining NCOA3 and FOXA1 binding strength by ChIP-qPCR (Supplemental Fig. 10). The control confirms what we found in the ChIP-seq data: FOXA1 binding does not significantly change on E2 stimulation and there is NCOA3 loss (Fig. 5E). In the NCOA3 overexpression experiment, however, we find no significant change in either FOXA1 or NCOA3 binding on E2 stimulation (Fig. 5F). We also examined the effect of NCOA3 overexpression on the expression of five genes downregulated by estrogen and found that NCOA3 overexpression reduced the extent of these expression changes (Supplemental Fig. 11). These results are consistent with the physiological squelching mechanism in which E2-induced ESR1 binding sites compete with FOXA1 sites for the NCOA3 coregulator.

\section{Discussion}

Using genome-wide DNase-seq and H3K4me2 ChIP-seq analyses, we have mapped important features of enhancer-associated chromatin. We observed systematic differences in nucleosome occupancy patterns and DHS associated with different transcription factors in LNCaP and MCF-7 cell lines. While AR binding in LNCaP cells has large effects on nucleosome occupancy, ESR1 binding in MCF-7 cells is not strongly influenced by, nor does it influence, nucleosome occupancy. In LNCaP cells, it has been reported that a knockdown of FOXA1 expression causes a dramatic change in AR binding locations, including the gain of numerous sites that are not observed under normal FOXA1 conditions (Wang et al. 2011). Notably, these new AR binding sites were not associated with observable nucleosome remodeling but were more like the ESR1 binding we observed in MCF-7 cells.

Thermodynamic equilibrium has been proposed to explain experimentally observed genome-wide in vivo nucleosome occupancy patterns. In this model, both nucleosomes and transcription factors have an intrinsic affinity for DNA sequence that is dependent on sequence composition (Segal and Widom 2009). Transcription factors compete with nucleosomes for DNA, and thermodynamic equilibrium determines the configuration of nucleosomes and transcription factors. In addition, nucleosome occupancy is likely to be shaped by kinetic elements, in particular, chromatin-remodeling factors using the energy derived from ATP hydrolysis to actively modify DNA-histone interactions. The importance of ATP-dependent factors was demonstrated in a recent study that showed that ATP is essential for creating the strongly positioned nucleosome arrays observed near TSSs in Saccharomyces (Zhang et al. 2011). Experimental evidence shows that different chromatin remodeling enzymes are recruited to enhancer loci by sequence-specific transcription factors (Peterson and Workman 2000), such as nuclear receptors. For example, BRG-1, the active component of human SWI/SNF chromatin-remodeling complexes, has been shown to be a key factor that potentiates AR- and ESR1-regulated transcription (DiRenzo et al. 2000; Dai et al. 2008). Both AR and ESR1 are known to interact directly with BAF57, a component of the SWI/SNF remodeling complexes (Belandia et al. 2002; Link et al. 2005). Several modes of chromatin remodeling have been suggested, including nucleosome sliding, nucleosome eviction, and looping of DNA away from the histone core. We speculate that the distinct mechanisms of the different classes 
A

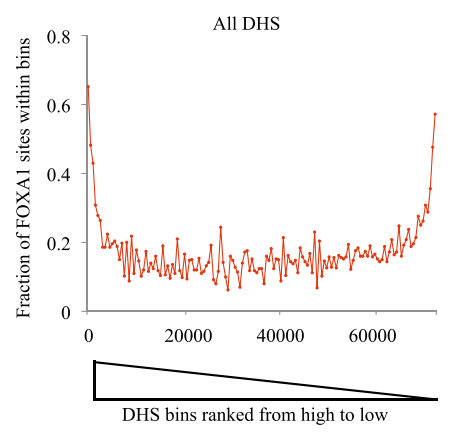

C

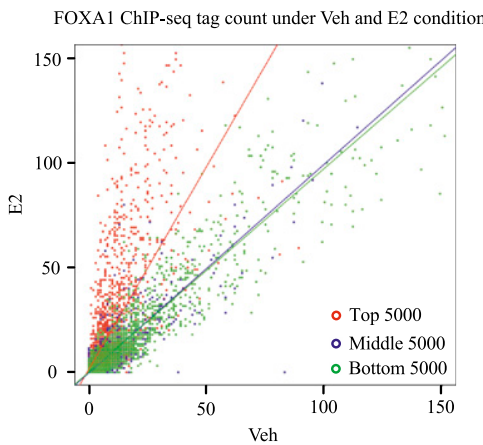

$\mathbf{E}$

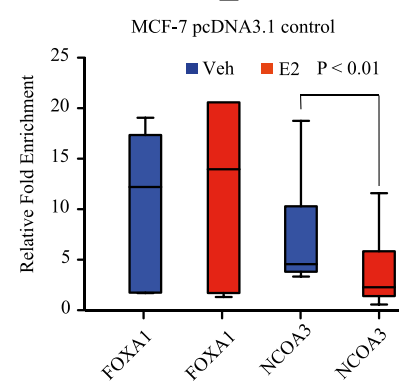

B

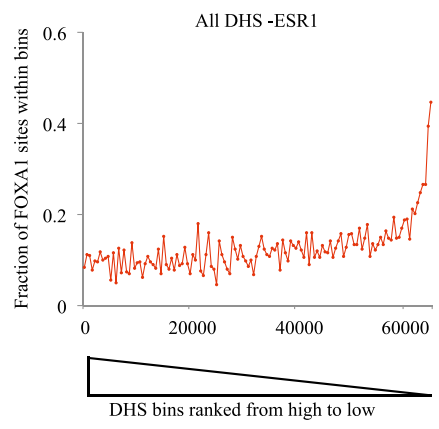

D

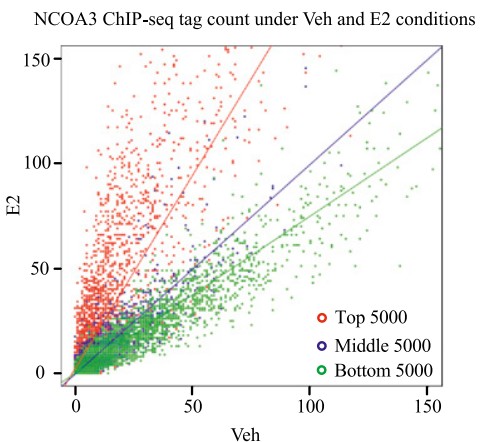

$\mathbf{F}$

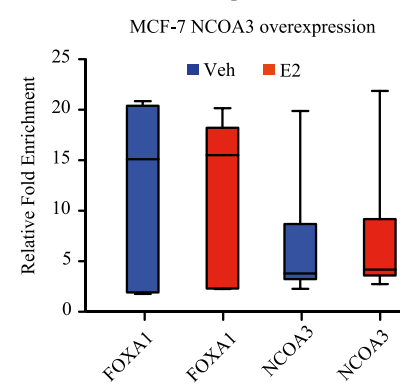

Figure 5. DNase I hypersensitivity changes at FOXA1 and NCOA3 sites. Association of $\triangle \mathrm{DHS}$ with FOXA1 sites in the presence $(A)$ and absence $(B)$ of ESR1 binding. MCF-7 DHS in the estrogen-stimulated condition were ranked in descending order based on the $\triangle \mathrm{DHS}$ score. These ranked regions are grouped into bins of 500. ( $Y$-axis) Fraction of regions that overlap with FOXA1 ChIP-seq enriched regions. Scatter plots of FOXA1 $(C)$ and NCOA3 $(D)$ ChIP-seq tag counts in the stimulated condition compared with counts in the unstimulated condition. Three groups of 5000 DHS sites were selected from the MCF-7 estrogen-stimulated DHS sites: DHS-increased (red), DHS-unchanged (blue), and DHS-diminished (green). Regression lines were drawn for each of the groups. The steeper the slope of a regression line, the greater the binding of the factor in the E2-stimulated condition relative to the unstimulated condition. While the slope for FOXA1 in the DHS-diminished category is not significantly different from that in the DHS-unchanged category, the slope for NCOA3 in the DHS-diminished category is less than that for the DHS-unchanged category. This means that within the DHS-diminished category NCOA3 binding tends to decrease on E2 stimulation while FOXA1 binding is maintained at the same level. Changes of FOXA1 and NCOA3 binding strength at FOXA1 binding sites in the overexpression control $(E)$ and NCOA3 overexpression $(F)$ samples under stimulated and unstimulated conditions. Six FOXA1 binding sites were selected from the hormone-diminished DHS sites. Box plots were generated from the ChIP-qPCR data of the six sites tested. The individual ChIP-qPCR assays are shown in Supplemental Figure 10.

of ATP-dependent remodeling enzymes may explain the differential chromatin effects seen in our experiments. Our study demonstrates how MNase digestion and DHS chromatin assays provide complementary information on chromatin structure.
Our differential DNase I hypersensitivity experiments revealed a surprising link between coregulator and chromatin structure. Significantly, this link was not merely a consequence of FOXA1 binding itself. NCOA3 ChIP-seq in MCF-7 cells under vehicle and estrogen-induced conditions revealed that, although a high overlap between NCOA3 and ESR1 was observed, an unexpectedly high overlap between FOXA1 binding sites and NCOA3enriched loci was also found (Lanz et al. 2010). Previously, coregulators and chromatin remodeling activity had been shown to act synergistically in the AR and ESR1 systems in collaboration with the AR and ESR1 factors themselves (Metivier et al. 2003; Wang et al. 2005). Here, we find evidence for a chromatin remodelingcoregulator synergy that is associated with FOXA1 in the absence of ESR1 or AR. Our experiment supports the hypothesis that physiological squelching is an important mechanism involved in the down-regulation of genes at early time points following estrogen treatment.

According to our current understanding, DNase I hypersensitivity occurs in nucleosome free regions that are close to transcription factor binding sites. Although we do observe many DHS in nonnucleosomal DNA, DHS sites sometimes occur in regions having high nucleosome occupancy. In particular, we identified a set of DHS sites that were associated with ESR1 binding to nucleosomal DNA. The different nucleosome occupancy and DNase I hypersensitivity patterns that we observed are likely dependent on not only the details of the transcription factor-DNA interaction but also on the chromatin environment at the binding site. Relevant aspects of the chromatin environment may include post-translational histone modifications, the composition of the nucleosomes themselves, and the presence of other protein complexes. Histone post-translational modifications may influence transcription factor binding by enhancing the affinity of transcription factor related protein complexes for the modified histone or by reducing the affinity of the histone octamer for DNA. The structure of the nucleosome cores may also determine nucleosomes as being more or less permissive to transcription factor binding as histones that constitute nucleosomes come in variants, such as H2A.Z, that have been reported to alter nucleosome properties (Jin et al. 2009).

In our analysis of genome-wide dynamic DNase-seq, we noted three important factors that contribute to DNase I hypersensitivity. 
First, in agreement with the standard view, the majority of DHS sites occur in nucleosome-free regions. Second, DHS frequently arises as a result of transcription factor binding; however, they do not necessarily occur in nucleosome-free regions. Third, DHS can change with the addition or removal of cofactors. We demonstrated that dynamic DNase-seq is an effective and informative approach that can be used to locate enhancers that regulate a cell's transcriptional response to stimuli.

\section{Methods}

\section{Cell line and culture conditions}

The prostate cancer cell line LNCaP was obtained from the American Type Culture Collection. LNCaP cells were maintained in RPMI 1640 medium supplemented with $10 \%$ fetal bovine serum (FBS), $2 \mathrm{mM}$ glutamine, $100 \mathrm{U} / \mathrm{mL}$ penicillin, and $100 \mathrm{mg} / \mathrm{mL}$ streptomycin. The hormone-independent breast cancer cell line MCF-7:2A and the parental MCF-7 cell line were from V. Craig Jordan's lab. MCF-7 cells were maintained in RPMI 1640 medium supplemented with $10 \%$ fetal bovine serum (FBS), $2 \mathrm{mM}$ glutamine, $100 \mathrm{U} / \mathrm{mL}$ penicillin, $100 \mathrm{mg} / \mathrm{mL}$ streptomycin, $1 \times$ NEAA, and $6 \mu \mathrm{g} / \mathrm{L}$ insulin. MCF-7:2A cells were maintained in phenolred-free RPMI 1640 medium supplemented with 10\% charcoal stripped FBS. LNCaP and MCF-7 cells were starved in phenol-redfree medium supplemented with $10 \%$ charcoal stripped FBS for $3 \mathrm{~d}$ before hormone stimulation.

\section{ChIP and ChIP-seq}

The ChIP experiments were performed as previously described (He et al. 2010). We used antibodies to ESR1 (Ab-10 from Neomarkers; HC-20 from Santa Cruz), AR (N-20 from Santa Cruz), FOXA1 (ab23738 from Abcam), and H3K4me2 (07-030 from Millipore). Library construction was performed using the Illumina ChIP-seq DNA sample Prep Kit according to the manufacture's instruction; the libraries were sequenced at a length of $35 \mathrm{bp}$ with the Illumina Genome Analyzer. Model-based Analysis of ChIP-seq (MACS) software (Zhang et al. 2008a) was used to detect ChIP-seq peak regions. Nucleosome Positioning from Sequencing (NPS) software (Zhang et al. 2008b) was used to identify nucleosome positions based on the H3K4me2 ChIP-seq data. Binding Inference from Nucleosome Occupancy Changes (BINOCh) software (Meyer et al. 2011) was used to predict transcription factor binding events from the H3K4me2 NPS data.

\section{DNase hypersensitivity mapping}

DNase hypersensitivity mapping was performed as previously described with brief modifications (Ling et al. 2010; John et al. 2011). LNCaP cells were starved for $3 \mathrm{~d}$ in phenol-red-free medium supplemented with $10 \%$ charcoal stripped FBS and then treated with ethanol or active androgen $5 \alpha$-dihydrotestosterone (DHT) at a final concentration of $10 \mathrm{nM}$ for $4 \mathrm{~h}$. MCF-7 cells were starved the same way and then treated with ethanol or $17 \beta$-estrodial (E2) at a final concentration of $10 \mathrm{nM}$ for $45 \mathrm{~min}$. The cells were trypsinized and pelleted prior to washing and resuspension in buffer $\mathrm{A}$ (15 mM Tris-Cl [pH 8.0], $15 \mathrm{mM} \mathrm{NaCl}, 60 \mathrm{mM} \mathrm{KCl,} 1 \mathrm{mM}$ EDTA [pH 8.0], 0.5 mM EGTA [pH 8.0], 0.5 mM spermidine, and $0.15 \mathrm{mM}$ spermine) to a final concentration of $2 \times 10 \mathrm{M}$ cells $/ \mathrm{mL}$. Nuclei were extracted by adding buffer A containing NP-40. The nuclei were washed with buffer $\mathrm{A}$ and resuspended in prewarmed lysis buffer (13.5 mM Tris-HCl [pH 8.0], $87 \mathrm{mM} \mathrm{NaCl}, 54 \mathrm{mM} \mathrm{KCl}, 6 \mathrm{mM}$ $\mathrm{CaCl} 2,0.9 \mathrm{mM}$ EDTA, $0.45 \mathrm{mM}$ EGTA) at a concentration of $5 \mathrm{M} / \mathrm{mL}$ and then digested with different amounts of DNase I (Roche, 0-75
$\mathrm{U}$ ) for $5 \mathrm{~min}$ at $37^{\circ} \mathrm{C}$. The reactions were terminated by the addition of an equal volume of stop buffer ( $1 \mathrm{M}$ Tris- $\mathrm{Cl}$ [pH 8.0], $5 \mathrm{M} \mathrm{NaCl}$, $20 \%$ SDS, $0.5 \mathrm{M}$ EDTA [pH 8.0], and $10 \mu \mathrm{g} / \mathrm{mL}$ of RNase A [Roche]) and incubated at $55^{\circ} \mathrm{C}$. After $15 \mathrm{~min}$, Proteinase $\mathrm{K}$ (final concentration of $20 \mu \mathrm{g} / \mathrm{mL}$ ) was added to each digestion reaction and incubated for $2 \mathrm{~h}$ at $55^{\circ} \mathrm{C}$. DNA was extracted by careful phenolchloroform purification. The isolated DNA was run out on a gel, and DNA fragments between 100 and 400 bp long were gel-selected. The libraries were prepared following the Illumina library preparation protocol. DNase-seq libraries were sequenced at the Beijing Genomic Institute and the Center for Cancer Computational Biology (CCCB) at the Dana-Farber Cancer Institute.

\section{NCOA3 overexpression experiments}

A total of $12 \mu \mathrm{g}$ of pcDNA3.1-NCOA3 construct or the control empty vector were transfected in MCF-7 cells in 10-cm culture dishes using lipofectamine 2000 (Invitrogen) according to the manufacturer's instructions. After $72 \mathrm{~h}$ of transfection, cells were treated with estrogen or ethanol control for $45 \mathrm{~min}$ and then processed for ChIP-qPCR. For RT-qPCR, $3 \mu \mathrm{g}$ of the pcDNA3.1NCOA3 or the empty vector were transfected in MCF-7 cells in sixwell plates. After $72 \mathrm{~h}$ of transfection, cells were treated with estrogen or ethanol control for $3 \mathrm{~h}$. RNA was isolated using RNeasy mini kit (Qiagen) following the manufacturer's instructions. PCR primers used in this work are listed in Supplemental Table 1.

\section{Model for identifying differential DNase I hypersensitivity locations}

DNase I hypersensitive regions were identified using MACS with the default parameters. A tag was considered to belong to a genomic interval if, when shifted $100 \mathrm{bp}$ in a strand-directed direction, the entire tag fell within that interval. Each peak $i$ from the set of $m$ MACS peaks was then given a DHS change score $(\Delta \mathrm{DHS})$ by the formula:

$$
\Delta D H S_{i}=\sqrt{n_{i}^{\text {treat }} /\left(\sum_{k=1}^{m} n_{k}^{\text {treat }}\right) / m}-\sqrt{n_{i}^{\text {control }} /\left(\sum_{k=1}^{m} n_{k}^{\text {control }}\right) / m} .
$$

In this formula, $n_{i}$ is the tag count in a 600 -bp interval centered on the $i$-th MACS peak. The superscripts treat and control refer to the hormone-stimulated and vehicle conditions, respectively. We use the square root transformation to stabilize the variance of the score, allowing regions with high counts to be compared with those having low counts. Peaks within $1 \mathrm{~kb}$ of any RefSeq TSS were excluded from all analyses so as not to confound transcription factor binding effects with transcriptional ones. All analyses involving motifs enriched in the peak regions were identified using the BINOCh motif analysis software.

\section{Precision recall analysis}

To evaluate the ability of our method to predict TF binding we defined a set of bound and unbound genomic locations. We defined the bound set as the summits of MACS peaks determined from ChIP-seq data and located $>1 \mathrm{~kb}$ from the nearest RefSeq TSS. To define the unbound set, we downloaded a file of "mappable" genomic locations, "wgEncodeCrgMapabilityAlign50mer.bw.gz" from http://hgdownload.cse.ucsc.edu/goldenPath/hg18/encodeDCC/ wgEncodeMapability/ and selected a set of 850,000 non-bound, non-TSS sites by randomly sampling genomic locations that had a mappability index $>0.9$. These locations were filtered to not lie within $1 \mathrm{~kb}$ of any RefSeq TSS, TF ChIP-seq summit or other random location. The background was then scaled up to cover $2 \mathrm{~Gb}$, 
the size of the mappable genome. A DHS or ChIP-seq region was considered to be a true positive if its center was within $250 \mathrm{bp}$ of a TF summit and a false positive if its center was within $250 \mathrm{bp}$ of a background site. For motif analysis $200 \mathrm{bp}$ from the center of the DHS or ChIP-seq region was scanned using the BINOCh software (Meyer et al. 2011). CENTIPEDE predictions (Pique-Regi et al. 2011) for ESR1 binding in MCF-7 were downloaded from http:// centipede.uchicago.edu/SimpleMulti/. In the performance evaluation CENTIPEDE predictions were treated the same way as our DHS regions. Since the result we retrieved from the website contains no scoring information for the sites predicted by CENTIPEDE, a single point was drawn for the performance evaluation.

\section{DHS boxplots}

Tag counting under DHS peaks was carried out as before. Peaks were considered to be overlapping if their summits were within $600 \mathrm{bp}$ of each other. Box plots were produced using R with default parameters. The outliers beyond the whiskers are not shown. The $P$-values were calculated using the Mann-Whitney test.

\section{Gene expression data}

Affymetrix U133 Plus 2.0 microarray data (GSE7868) (Wang et al. 2007) in LNCaP cells and the processed GRO-seq gene expression data (GSE27463) (Hah et al. 2011) in MCF-7 cells were used in this study. The microarray data were analyzed using the RMA algorithm (Irizarry et al. 2003) using a custom CDF probe (v11) mapping to the RefSeq genes (Dai et al. 2005). The statistical significance was calculated using limma software (Smyth and Speed 2003).

\section{Data access}

MCF-7 H3K4me2 ChIP-seq, LNCaP, and MCF-7 DNase-seq raw sequence tags, and processed bed files have been submitted to the NCBI Gene Expression Omnibus (GEO) (http://www.ncbi.nlm.nih. gov/geo) under accession number GSE33216.

\section{Acknowledgments}

We thank Dr. David J. Waxman and Dr. Guoyu Ling for help with the DNase-seq protocol. We thank Dr. Jason D. Lieb and Dr. Piotr Mieczkowski for help with the high-throughput sequencing. This work was supported by grants from the National Institutes of Health (1R01 GM099409 to X.S.L.; 2P50 CA090381-06 to C.A.M. and M.B.; 2R01 DK074967-06 to M.B. and X.S.L.; P30 CA051008 to V.C.J), the Mazzon Award (GM099409 to X.S.L.), the Department of Defense (W81XWH-10-1-0557 to H.H.H.), and the Prostate Cancer Foundation (to M.B.).

Author contributions: H.H.H., C.A.M., X.S.L., and M.B. designed the experiments and wrote the manuscript. V.C.J. provided the hormone-resistant breast cancer cells and revised the manuscript. H.H.H. and M.W.C. performed the experiments. C.A.M. and H.H.H. conducted the data analysis.

\section{References}

Ariazi EA, Cunliffe HE, Lewis-Wambi JS, Slifker MJ, Willis AL, Ramos P, Tapia C, Kim HR, Yerrum S, Sharma CGN, et al. 2011. Estrogen induces apoptosis in estrogen deprivation-resistant breast cancer through stress responses as identified by global gene expression across time. Proc Natl Acad Sci 108: 18879-18886.

Barski A, Cuddapah S, Cui K, Roh TY, Schones DE, Wang Z, Wei G, Chepelev I, Zhao K. 2007. High-resolution profiling of histone methylations in the human genome. Cell 129: 823-837.
Belandia B, Orford RL, Hurst HC, Parker MG. 2002. Targeting of SWI/SNF chromatin remodelling complexes to estrogen-responsive genes. $E M B O$ I 21: 4094-4103.

Carroll JS, Liu XS, Brodsky AS, Li W, Meyer CA, Szary AJ, Eeckhoute J, Shao W, Hestermann EV, Geistlinger TR, et al. 2005. Chromosome-wide mapping of estrogen receptor binding reveals long-range regulation requiring the forkhead protein FoxA1. Cell 122: 33-43.

Carroll JS, Meyer CA, Song J, Li W, Geistlinger TR, Eeckhoute J, Brodsky AS, Keeton EK, Fertuck KC, Hall GF, et al. 2006. Genome-wide analysis of estrogen receptor binding sites. Nat Genet 38: 1289-1297.

Dai M, Wang P, Boyd AD, Kostov G, Athey B, Jones EG, Bunney WE, Myers RM, Speed TP, Akil H, et al. 2005. Evolving gene/transcript definitions significantly alter the interpretation of GeneChip data. Nucleic Acids Res 33: e175. doi: 10.1093/nar/gni179.

Dai Y, Ngo D, Jacob J, Forman LW, Faller DV. 2008. Prohibitin and the SWI/ SNF ATPase subunit BRG1 are required for effective androgen antagonist-mediated transcriptional repression of androgen receptorregulated genes. Carcinogenesis 29: 1725-1733.

DiRenzo J, Shang Y, Phelan M, Sif S, Myers M, Kingston R, Brown M. 2000. BRG-1 is recruited to estrogen-responsive promoters and cooperates with factors involved in histone acetylation. Mol Cell Biol 20: 75417549.

Hah N, Danko CG, Core L, Waterfall JJ, Siepel A, Lis JT, Kraus WL. 2011. A rapid, extensive, and transient transcriptional response to estrogen signaling in breast cancer cells. Cell 145: 622-634.

He HH, Meyer CA, Shin H, Bailey ST, Wei G, Wang Q, Zhang Y, Xu K, Ni M, Lupien M, et al. 2010. Nucleosome dynamics define transcriptional enhancers. Nat Genet 42: 343-347.

Hu G, Schones DE, Cui K, Ybarra R, Northrup D, Tang Q, Gattinoni L, Restifo NP, Huang S, Zhao K. 2011. Regulation of nucleosome landscape and transcription factor targeting at tissue-specific enhancers by BRG1. Genome Res 21: 1650-1658.

Irizarry RA, Hobbs B, Collin F, Beazer-Barclay YD, Antonellis KJ, Scherf U, Speed TP. 2003. Exploration, normalization, and summaries of high density oligonucleotide array probe level data. Biostatistics 4: 249264 .

Jin C, Zang C, Wei G, Cui K, Peng W, Zhao K, Felsenfeld G. 2009. H3.3/ H2A.Z double variant-containing nucleosomes mark 'nucleosome-free regions' of active promoters and other regulatory regions. Nat Genet 41: 941-945.

John S, Sabo PJ, Thurman RE, Sung MH, Biddie SC, Johnson TA, Hager GL, Stamatoyannopoulos JA. 2011. Chromatin accessibility pre-determines glucocorticoid receptor binding patterns. Nat Genet 43: 264-268.

Joseph R, Orlov YL, Huss M, Sun W, Kong SL, Ukil L, Pan YF, Li G, Lim M, Thomsen JS, et al. 2010. Integrative model of genomic factors for determining binding site selection by estrogen receptor- $\alpha$. Mol Syst Biol 6: 456. doi: $10.1038 / \mathrm{msb} .2010 .109$.

Lanz RB, Bulynko Y, Malovannaya A, Labhart P, Wang L, Li W, Qin J, Harper M, O'Malley BW. 2010. Global characterization of transcriptional impact of the SRC-3 coregulator. Mol Endocrinol 24: 859-872.

Ling G, Sugathan A, Mazor T, Fraenkel E, Waxman DJ. 2010. Unbiased, genome-wide in vivo mapping of transcriptional regulatory elements reveals sex differences in chromatin structure associated with sexspecific liver gene expression. Mol Cell Biol 30: 5531-5544.

Link KA, Burd CJ, Williams E, Marshall T, Rosson G, Henry E, Weissman B, Knudsen KE. 2005. BAF57 governs androgen receptor action and androgen-dependent proliferation through SWI/SNF. Mol Cell Biol 25: 2200-2215.

Lupien M, Eeckhoute J, Meyer CA, Wang Q, Zhang Y, Li W, Carroll JS, Liu XS, Brown M. 2008. FoxA1 translates epigenetic signatures into enhancerdriven lineage-specific transcription. Cell 132: $958-970$.

Metivier R, Penot G, Hubner MR, Reid G, Brand H, Kos M, Gannon F. 2003. Estrogen receptor- $\alpha$ directs ordered, cyclical, and combinatorial recruitment of cofactors on a natural target promoter. Cell 115: 751763.

Meyer ME, Gronemeyer H, Turcotte B, Bocquel MT, Tasset D, Chambon P. 1989. Steroid hormone receptors compete for factors that mediate their enhancer function. Cell 57: 433-442.

Meyer CA, He HH, Brown M, Liu XS. 2011. BINOCh: Binding inference from nucleosome occupancy changes. Bioinformatics 27: 1867-1868.

Peterson CL, Workman JL. 2000. Promoter targeting and chromatin remodeling by the SWI/SNF complex. Curr Opin Genet Dev 10: $187-192$.

Pink JJ, Jiang SY, Fritsch M, Jordan VC. 1995. An estrogen-independent MCF-7 breast cancer cell line which contains a novel 80-kilodalton estrogen receptor-related protein. Cancer Res 55: 2583-2590.

Pique-Regi R, Degner JF, Pai AA, Gaffney DJ, Gilad Y, Pritchard JK. 2011. Accurate inference of transcription factor binding from DNA sequence and chromatin accessibility data. Genome Res 21: 447-455.

Segal E, Widom J. 2009. Poly(dA:dT) tracts: Major determinants of nucleosome organization. Curr Opin Struct Biol 19: 65-71. 
Siersbaek R, Nielsen R, John S, Sung MH, Baek S, Loft A, Hager GL, Mandrup S. 2011. Extensive chromatin remodelling and establishment of transcription factor 'hotspots' during early adipogenesis. EMBO J 30: 1459-1472.

Smyth GK, Speed T. 2003. Normalization of cDNA microarray data. Methods 31: $265-273$.

Song L, Zhang Z, Grasfeder LL, Boyle AP, Giresi PG, Lee BK, Sheffield NC, Graf S, Huss M, Keefe D, et al. 2011. Open chromatin defined by DNaseI and FAIRE identifies regulatory elements that shape cell-type identity. Genome Res 21: 1757-1767.

Torchia J, Rose DW, Inostroza J, Kamei Y, Westin S, Glass CK, Rosenfeld MG. 1997. The transcriptional co-activator p/CIP binds CBP and mediates nuclear-receptor function. Nature 387: 677-684.

Verzi MP, Shin H, He HH, Sulahian R, Meyer CA, Montgomery RK, Fleet JC, Brown M, Liu XS, Shivdasani RA. 2010. Differentiation-specific histone modifications reveal dynamic chromatin interactions and partners for the intestinal transcription factor CDX2. Dev Cell 19: 713-726.

Wang Q, Carroll JS, Brown M. 2005. Spatial and temporal recruitment of androgen receptor and its coactivators involves chromosomal looping and polymerase tracking. Mol Cell 19: 631-642.

Wang Q, Li W, Liu XS, Carroll JS, Janne OA, Keeton EK, Chinnaiyan AM, Pienta KJ, Brown M. 2007. A hierarchical network of transcription factors governs androgen receptor-dependent prostate cancer growth. Mol Cell 27: 380-392.
Wang Q, Li W, Zhang Y, Yuan X, Xu K, Yu J, Chen Z, Beroukhim R, Wang H, Lupien $M$, et al. 2009. Androgen receptor regulates a distinct transcription program in androgen-independent prostate cancer. Cell 138: $245-256$.

Wang D, Garcia-Bassets I, Benner C, Li W, Su X, Zhou Y, Qiu J, Liu W, Kaikkonen MU, Ohgi KA, et al. 2011. Reprogramming transcription by distinct classes of enhancers functionally defined by eRNA. Nature 474: 390-394.

Wu C. 1980. The $5^{\prime}$ ends of Drosophila heat shock genes in chromatin are hypersensitive to DNase I. Nature 286: 854-860.

Zhang Y, Liu T, Meyer CA, Eeckhoute J, Johnson DS, Bernstein BE, Nussbaum C, Myers RM, Brown M, Li W, et al. 2008a. Model-based analysis of ChIP-Seq (MACS). Genome Biol 9: R137. doi: 10.1186/gb2008-9-9-r137.

Zhang Y, Shin H, Song JS, Lei Y, Liu XS. 2008b. Identifying positioned nucleosomes with epigenetic marks in human from ChIP-Seq. BMC Genomics 9: 537. doi: 10.1186/1471-2164-9-537.

Zhang Z, Wippo CJ, Wal M, Ward E, Korber P, Pugh BF. 2011. A packing mechanism for nucleosome organization reconstituted across a eukaryotic genome. Science 332: 977-980.

Received October 12, 2011; accepted in revised form March 6, 2012. 


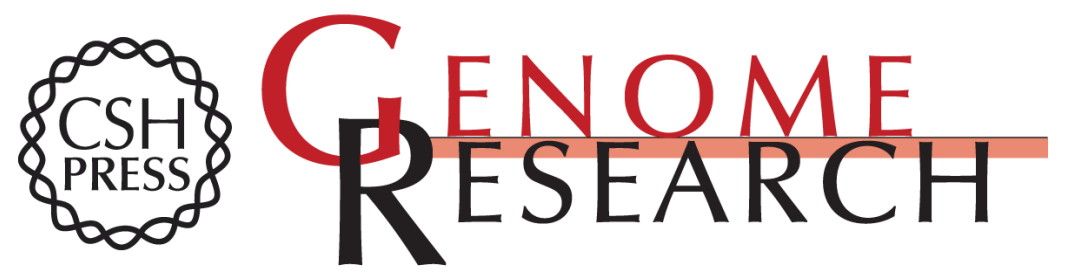

\section{Differential DNase I hypersensitivity reveals factor-dependent chromatin dynamics}

Housheng Hansen He, Clifford A. Meyer, Mei Wei Chen, et al.

Genome Res. 2012 22: 1015-1025 originally published online April 16, 2012

Access the most recent version at doi:10.1101/gr.133280.111

Supplemental Material

References

Creative

Commons

License

Email Alerting

Service
http://genome.cshlp.org/content/suppl/2012/03/19/gr.133280.111.DC1

This article cites 39 articles, 10 of which can be accessed free at: http://genome.cshlp.org/content/22/6/1015.full.html\#ref-list-1

This article is distributed exclusively by Cold Spring Harbor Laboratory Press for the first six months after the full-issue publication date (see

http://genome.cshlp.org/site/misc/terms.xhtml). After six months, it is available under a Creative Commons License (Attribution-NonCommercial 3.0 Unported License), as described at http://creativecommons.org/licenses/by-nc/3.0/.

Receive free email alerts when new articles cite this article - sign up in the box at the top right corner of the article or click here.

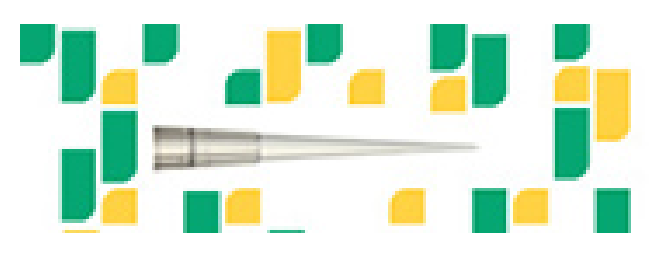

Focused on your science.

Jコగ

SCIENTIFIC

saos or seisnes

To subscribe to Genome Research go to: https://genome.cshlp.org/subscriptions 